\section{Salt-sensitive hypertension: if only it were as simple as rocket science}

\author{
Timothy L. Reudelhuber \\ Clinical Research Institute of Montreal (IRCM), Montreal, Quebec, Canada \\ J. Clin. Invest. 111:1115-1116 (2003). doi:10.1172/JCI200318397.
}

What? Salt and hypertension? Again? If your attention drifted and you haven't been able to follow the debate for the last 30 years, I'd highly recommend reading the 1998 Science article appropriately entitled "The (political) science of salt" (1). Both the original article and the heated responses it generated are more entertaining than a ringside seat at a Saturday night wrestling match. Why can't we agree on whether dietary salt is good or bad for people (or neither)? Some have suggested that the difficulty lies in the fact that the general population should be subclassified as salt "responders" and "non-responders". Another possibility, strengthened by the work of $\mathrm{Ni}$ et al. (2) in this issue of the JCI, is that additional hormonal factors modulate our salt sensitivity, making it a moving target.

\section{A tough nut to crack}

Hormonal modulation of salt balance and blood volume is an indisputable truth in physiology. But does it play a critical role in hypertension? Genetic evidence certainly supports the possibility that it can. In humans, rare genetic disorders of salt transport in the kidney have been shown to be responsible

Address correspondence to: Timothy L. Reudelhuber, Clinical Research Institute of Montreal (IRCM), 110 Pine Avenue West, Montreal, Quebec H2W 1R7, Canada.

Phone: (514) 987-5716; Fax: (514) 987-5717; E-mail: reudelt@ircm.qc.ca.

Conflict of interest: The author has declared that no conflict of interest exists.

Nonstandard abbreviations used: $\gamma$-melanocyte-stimulating hormone $(\gamma$-MSH); proopiomelanocortin (POMC); prohormone convertase (PC); melanocortin receptor (MC-R).

Figure 1 for several forms of hyper- and hypotension including Liddle, Bartter, and Gitelman syndromes (reviewed in ref. 3). Nevertheless, there's no clear evidence to date that the same genes that cause these disorders play a significant role in essential hypertension. But wait, maybe we just need to do a better job of subclassifying patients and regulating their diets when we do genetic screens. As an alternative and presumably simpler approach, animal models have been used. In the early 1960s, Dahl and colleagues reported that they had bred rats characterized by a salt-sensitive form of hypertension (4). These rats have been the subject of hundreds of research studies aimed at defining the genes and proteins responsible for salt-sensitivity. In spite of the fact that genetic heterogeneity and diet can be taken out of the equation with this approach, as of 3 years ago 24 chromosomal regions spread over 19 chromosomes had been found to contribute to hypertension in rats (5), with at least 8 of these regions having effects on blood pressure in the Dahl salt-sensitive strain. It now seems clear that although this approach still holds promise to uncover new targets in hypertension, it's anything but simple. This is where the ability to knockout genes in mice comes in handy. In the current issue, $\mathrm{Ni}$ et al. show that mice having an impaired ability to make $\gamma$-melanocyte-stimulating hormone $(\gamma-\mathrm{MSH})$ exhibit salt-sensitive hypertension (2).

$\gamma$-MSH is a small peptide hormone that is clipped out of the middle of the larger protein precursor proopiomelanocortin (POMC) by prohormone convertase 2 (PC2) (Figure 1). Expression of POMC in the pituitary gland results in the release of several hormones, including the melanocortins, into the circulation while its expression in the hypothalamus and brainstem leads to production of melanocortinergic neurotransmitters. In normal mice, $\gamma$-MSH increases in the circulation when the animals are placed on a highsalt diet. In contrast, in PC2-knockout mice $\gamma$-MSH does not increase and the mice develop hypertension. Of course this alone would not constitute strong evidence for the role of $\gamma$-MSH since PC2 is involved in generating a lot of other peptide and protein hormones

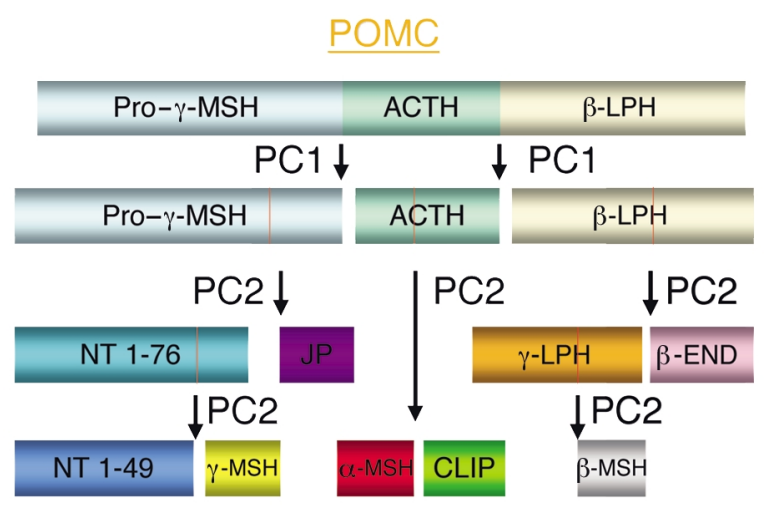

Schematic representation of the cleavage of pituitary proopiomelanocortin (POMC) by prohormone convertase (PC) enzymes 1 and 2 . Generated peptides include $\alpha-, \beta$-, and $\gamma$-melanocyte-stimulating hormone $(\mathrm{MSH}), \beta$ - and $\gamma$ - lipotropic hormone $(\mathrm{LPH})$, adrenocorticotropic hormone $(A C T H)$, $\beta$-endorphin ( $\beta$-END), and the amino-terminal (NT), joining (JP), and CLIP peptides. Note that the release of $\gamma$-MSH requires a cleavage by $\mathrm{PC} 2$ at both ends. 
including insulin, glucagon, and somatostatin. However, the authors also show that the hypertension seen in the high-salt-fed PC2-knockout animals can be corrected by administration of a synthetic $\gamma$-MSH analogue and that the same results are seen in mice in which the $\gamma$-MSH receptor, melanocortin receptor 3 (MC3-R), is knocked out. So why hasn't $\gamma$-MSH been implicated in salt-sensitive hypertension until now? Part of the reason is certainly the multitude of neuroendocrine signals mediated by the other peptides produced along with $\gamma$-MSH when POMC is processed (Figure 1), making it hard to isolate $\gamma$-MSH-specific actions in a physiological setting. In fact, 5 receptors have been identified to date - MC1-R, MC2-R, MC3-R, MC4-R, and MC5-R - for the melanocortin hormones alone, with more possibly to come. These receptors have a broad tissue distribution and they mediate a range of physiological responses depending on their location. These include: (a) pigmentation (MC1-R); (b) modulation of corticosterone levels (MC2-R); (c) appetite suppression and metabolic activation (MC4-R); (d) thermoregulation and water repulsion (MC5-R); and (e) inflammation (MC1-R and MC3-R). It's easy to imagine that several of these responses might have masked or complicated the analysis of blood pressure effects of $\gamma-\mathrm{MSH}$.

Does this mean that $\gamma-\mathrm{MSH}$ is the cause of salt-induced hypertension in humans? Not by a long shot! The knockout of other mouse genes, including those for atrial natriuretic peptide (6) and its receptor (7), the prostaglandin receptor EP2 (8), and the bradykinin receptor (9), also leads to salt-sensitive hypertension. However, none of these as yet has been linked to the condition in humans. Moreover, the existence of $\gamma-\mathrm{MSH}$ in humans is still a matter of some debate. Nevertheless, the possibility that hormones with both central and peripheral actions like $\gamma$-MSH could cause our salt-sensitivity to fluctuate is an attrac- tive hypothesis to test in a field where salient explanations are scarce.

1. Taubes, G. 1998. The (political) science of salt Science. 281:898-901, 903-907.

2. Ni, X.-P., Pearce, D., Butler, A.A., Cone, R.D., and Humphreys, M.H. 2003. Genetic disruption of $\gamma$-melanocyte-stimulating hormone signaling leads to salt-sensitive hypertension in the mouse. J. Clin. Invest. 111:1251-1258. doi:10.1172/JCI200316993.

3. Lifton, R.P., Gharavi, A.G., and Geller, D.S. 1998 Molecular mechanisms of human hypertension. Cell. 281:545-556.

4. Dahl, L.K., Heine, M., and Tassinari, L. 1962. Role of genetic factors in susceptibility to experimental hypertension due to chronic excess salt ingestion. Nature. 194:480-482.

5. Rapp, J.P. 2000. Genetic analysis of inherited hypertension in the rat. Physiol. Rev. 80:135-172.

6. John, S.W., et al. 1995. Genetic decreases in atrial natriuretic peptide and salt-sensitive hypertension. Science. 267:679-681.

7. Oliver, P.M., et al. 1998. Natriuretic peptide receptor 1 expression influences blood pressures of mice in a dose-dependent manner. Proc. Natl. Acad. Sci. U. S. A. 95:2547-2551.

8. Kennedy, C.R., et al. 1999. Salt-sensitive hypertension and reduced fertility in mice lacking the prostaglandin EP2 receptor. Nat. Med. 5:217-220.

9. Alfie, M.E., Yang, X.P., Hess, F., and Carretero, O.A. 1996. Salt-sensitive hypertension in bradykinin B2 receptor knockout mice. Biochem. Biophys. Res. Commun. 224:625-630.

\section{Coordinated tumor immunity}

\section{Glenn Dranoff}

Department of Medical Oncology, Dana-Farber Cancer Institute and Department of Medicine, Brigham and Women's Hospital and Harvard Medical School, Boston, Massachusetts, USA

J. Clin. Invest. 111:1116-1118 (2003). doi:10.1172/JCI200318359.

The most important achievement of cancer immunology thus far may be the development of robust techniques for the identification of tumor antigens $(1,2)$. This work underlies our current understanding that cancer patients frequently generate specific cellular and humoral antitumor responses. Moreover, the expression of

\footnotetext{
Address correspondence to: Glenn Dranoff, Dana-Farber Cancer Institute, Dana 510E, 44 Binney Street, Boston, Massachusetts 02115, USA. Phone: (617) 632-5051; Fax: (617) 632-5167;

E-mail: glenn_dranoff@dfci.harvard.edu. Conflict of interest: The author has declared that no conflict of interest exists.
}

transformation-associated stress genes commonly provokes innate immune reactions (3). Together, these findings unveil a previously unsuspected breadth of immune recognition in tumor bearing hosts.

The characterization of cancer cell antigenicity has fueled efforts to delineate protective immune-effector mechanisms. The task is complicated by the dual role that immunity plays during cancer progression. Recent studies disclosed a marked increase in the incidence of spontaneous and chemicallyinduced tumors in immunodeficient mice compared to littermate controls (4). Since immunocompetent animals efficiently reject tumor transplants from immunodeficient hosts, the experiments support the idea that the immune system functions as an extrinsic tumor suppressor. Consistent with this concept, clinical-pathologic investigations established a strong association between the presence of dense intratumoral $\mathrm{T}$ cell infiltrates and favorable clinical outcomes in patients with malignant melanoma or carcinomas of the colon, kidney, and ovary $(5,6)$.

Other work indicates, though, that tumors may subvert the immune system to facilitate disease progression (7). Unresolved inflammation, whether due to infection, autoimmunity, or environmental agents, markedly increases the risk of cancer. Dysregulated cytokine production promotes cell proliferation and attenuates apoptosis. Phagocyte-derived reactive oxygen species damage DNA. Tumor cell invasion and metastasis exploit the normal cues for leukocyte migration. Collectively, these studies illustrate diverse ways in which the immune system sculpts the hallmarks of cancer (8). 